\title{
REGISTRO DE TERMITAS (Isoptera) ASOCIADAS A CULTIVOS DE MANGO (Mangifera indica) EN EL DEPARTAMENTO DEL MAGDALENA, COLOMBIA
}

\author{
RECORDS OF TERMITES (Isoptera) ASSOCIATED WITH MANGO CROPS \\ (Mangifera indica) IN THE DEPARTMENT OF MAGDALENA, COLOMBIA
}

\author{
Hafid Yesid Hurtado-Borrero (D, Diego Armando Manga-Candelario (D) y Paula Andrea Sepúlveda-Cano (D)
}

\begin{abstract}
RESUMEN
Las termitas (Isoptera) son insectos sociales que ocasionan daños en muchos cultivos de interés económico. Teniendo en cuenta la importancia del cultivo de mango en la economía de los agricultores del departamento del Magdalena y la expansión de este cultivo en la región, se realizó un inventario inicial de las especies de termitas presentes. Se realizaron muestreos en 22 huertos ubicados en las diferentes zonas productoras del departamento del Magdalena. Se realizó una recolección manual de los especímenes de termitas en cada zona y se determinaron dos familias: Rhinotermitidae, con un género (Heterotermes), y Termitidae, con dos géneros (Microcerotermes y Nasutitermes). Se identificaron cuatro morfoespecies, dentro de las cuales Nasutitermes sp. presentó la mayor abundancia y una amplia distribución en el área de estudio, seguida de N. nigriceps (Haldeman), Microcerotermes sp. y Heterotermes tenuis. Se requieren nuevas observaciones en campo y laboratorio que permitan determinar los factores que afectan en la distribución e incidencia de las especies de termitas en el departamento y un análisis sobre su impacto económico en el cultivo.
\end{abstract}

PALABRAS CLAVE: Nasutitermes; Rhinotermitidae; Termitidae

\begin{abstract}
Termites (Isoptera) are social insects that cause damage to many crops of economic interest. Given the importance of mango crops in the economy of the farmers in the department of Magdalena and the growth of this crop in the region, a preliminary termites inventory was carried out. Samplings were taken in 22 orchards located in the different producing areas of the department of Magdalena. Manual collections of termite specimens were made in each area and two families were determined: Rhinotermitidae, with one genus (Heterotermes), and Termitidae, with two genera (Microcerotermes, y Nasutitermes). Four morphospecies were identified. Nasutitermes sp. was the most frequent in the sampled orchards, followed by N. nigriceps (Haldeman), Microcerotermes sp. and Heterotermes tenuis. New field and laboratory observations aimed at determining the factors affecting the distribution and incidence of termite species in the department and an analysis of their economic impact on the crop are required.
\end{abstract}

KEY WORDS: Nasutitermes; Rhinotermitidae; Termitidae 


\section{INTRODUCCIÓN}

El mango (Mangifera indica) es uno de los cultivos de mayor proyección económica en la fruticultura del departamento del Magdalena; sin embargo, en todas las zonas donde se cultiva se presenta una alta incidencia de insectos plaga, ocasionando pérdidas que en ocasiones hacen insostenible el cultivo (Galán, 1999). Además de los daños directos, algunas lesiones ocasionadas por estos insectos pueden abrir un sitio de ingreso para agentes patógenos causantes de otros problemas fitosanitarios que afectan el rendimiento y la calidad del producto (Kondo-R, 2010). En Colombia, los principales insectos que afectan el cultivo de mango son los siguientes: mosca de la fruta (Diptera: Tephritidae), escamas y cochinillas (Hemiptera: Coccomorpha), trips (Thysanoptera), termitas (Isoptera), hormigas (Hymenoptera: Formicidae), chinches y pulgones (Hemiptera: Pentatomidae, Membracidae, Aphididae), y mariposas (Lepidoptera: Nymphalidae, Limacodidae, Megalopygidae) (Cartagena et al., 1992; Anacafé 2004; CORPOICA y Asohofrucol, 2013).

Dentro de estos artrópodos se destacan las termitas, insectos que viven en sociedades y han logrado aprovechar la lignocelulosa como recurso nutricional; se alimentan principalmente de las capas del cambium de las plantas, aunque actualmente usan otros sustratos alimenticios como hongos, pasto, humus y estiércol de ungulados (Krishna et al., 2013a). Para el caso de mango, el ataque de estos insectos reduce su crecimiento, principalmente por las dificultades en el desarrollo y establecimiento de raíces (Rogers et al., 1999), aunque algunos autores los consideran "plagas menores" de este cultivo (Hill, 2008).

En los cultivos de mango alrededor del mundo se han registrado varias especies de termitas asociadas, mostrando mayor susceptibilidad los árboles jóvenes (Bignell et al., 2011) y huertos localizados en zonas semi-áridas y secas (Abrol, 2015). Algunas de estas especies son Odontotermes spp. y Neotermes mangiferae en la India (Kannan y Venugopala, 2006; Krishna et al., 2013b), Macrotermes gilvus y Nasutitermes spp. en Filipinas (Acda, 2013), Ancistrotermes cavithorax en Senegal (Han y Ndiaye, 1996), Microtermes mycophagus en Pakistan (Iqbal et al., 2015) y Nasutitermes corniger, N. ephratae y N. nigriceps en Nicaragua (Maes, 2004). Para el caso de Colombia y en especial en la región Caribe, los trabajos más recientes en termitas se han realizado en cultivos de cítricos. En un inventario realizado en los departamentos de Atlántico, Bolívar, Cesar, Córdoba y Magdalena, Abadía et al. (2013) encontraron una incidencia de termitas, superior al 70 $\%$, siendo el departamento del Magdalena el de mayor afectación, con un porcentaje del 75,56 \% de incidencia, por lo que sugirieron realizar monitoreo y evaluaciones a las poblaciones de las especies más frecuentes. Sin embargo, la información para otros frutales de la región es escasa y se desconocen las especies de termitas asociadas. En este contexto, el presente trabajo buscó realizar un reconocimiento inicial de las especies asociadas a cultivos de mango de las zonas de mayor producción del frutal en el departamento del Magdalena.

\section{MATERIALES Y MÉTODOS}

Los muestreos se realizaron en 22 huertos productores de mango (con mínimo 0,5 ha) localizados en tres zonas del departamento del Magdalena: zona norte (municipios Santa Marta y Ciénaga), zona centro (municipio Pivijai) y zona sur (municipios San Sebastián y Guamal). Los huertos fueron heterogéneos en cuanto al manejo agronómico, edad de las plantas y variedades cultivadas, dentro de las que se destacaron los cultivares Azúcar (50 \%) e Hilaza (31 \%), mientras que el $19 \%$ restante tenían cultivadas las dos anteriores y mezclas de las variedades Manzano, Filipino, Bocado, Chupa, Tommy, Número 11 y Chancleta. En cada huerto se realizó un recorrido en zig-zag y se inspeccionaron aproximadamente el $10 \%$ de los árboles. En cada planta se examinaron la base del árbol, el tronco, las ramas, las hojas y los frutos, y ante la presencia de termitas se tomaron muestras de soldados, se depositaron en alcohol al $75 \%$ y se rotularon. En laboratorio se llevó a cabo el proceso de identificación, siguiendo las claves taxonómicas propuestas por Constantino (2002). Adicionalmente, en campo se tomaron los datos de número de árboles afectados y cantidad de ramas con galerías conspicuas. El material se depositó en el Centro de Colecciones Biológicas de la Universidad del Magdalena.

\section{RESULTADOS}

Se registraron daños por termitas en las cuatro zonas productoras del mango del departamento (Figura 1) y en el $77 \%$ de los huertos evaluados, con presencia de nidos tanto en la base como en la parte aérea de los árboles. Además, se encontraron túneles en los troncos y ramas y resquebrajamiento y secamiento de ramas. Tras la determinación taxonómica, se encontraron 
dos especies y dos morfoespecies distribuidas en tres géneros: Heterotermes tenuis (Hagen, 1858) (Rhinotermitidae), Microcerotermes sp. (Silvestri, 1901), Nasutitermes nigriceps (Haldeman) (Termitidae) y Nasutitermes sp. (Figura 2a-d). Las morfoespecies del

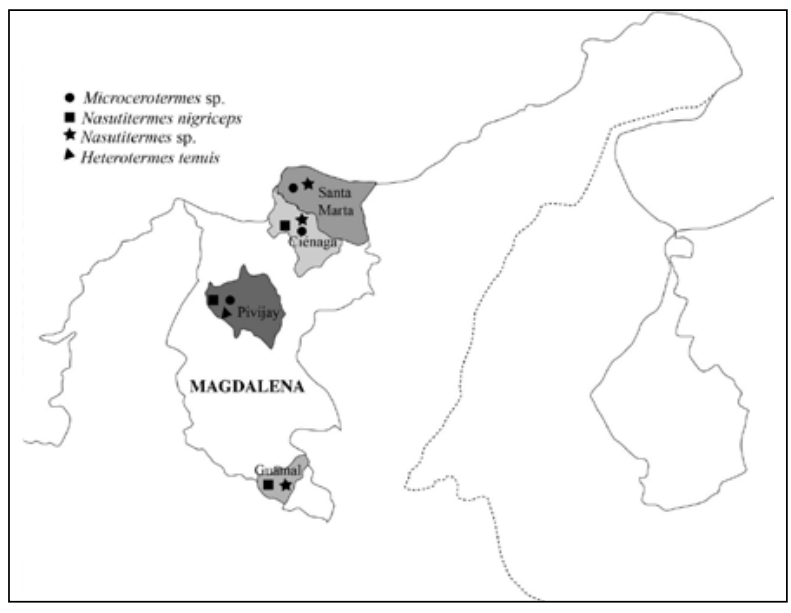

Figura 1. Distribución de las morfoespecies de termitas en las tres zonas productoras de mango del departamento. género Nasutitermes presentaron la mayor frecuencia de captura y la distribución geográfica más amplia, mientras que $H$. tenuis fue la especie con menor frecuencia de captura y la única que se restringió a la zona centro (Figuras 1 y 3 ).

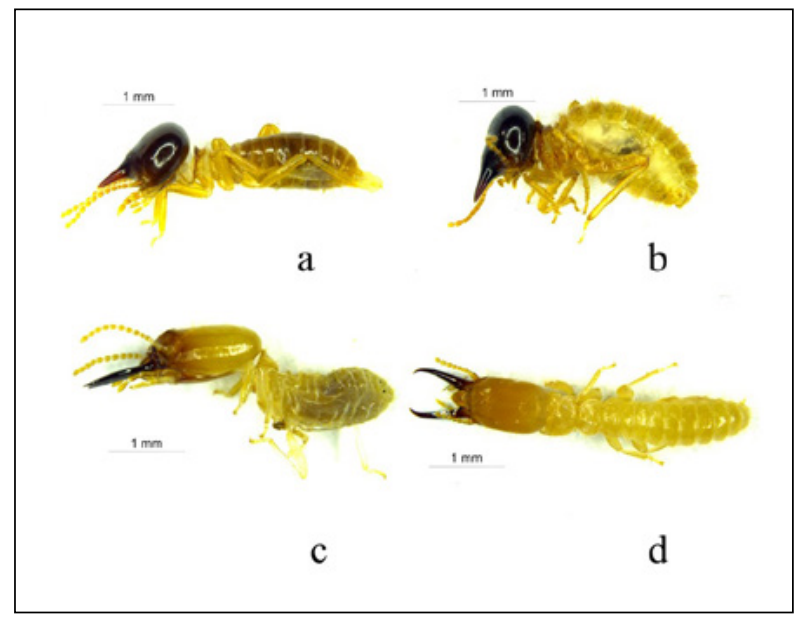

Figura 2. Morfoespecies de termitas encontradas asociadas al cultivo de mango del departamento del Magdalena a) Nasutitermes nigriceps b) Nasutitermes sp. c) Microcerotermes sp. d) Heterotermes tenuis.

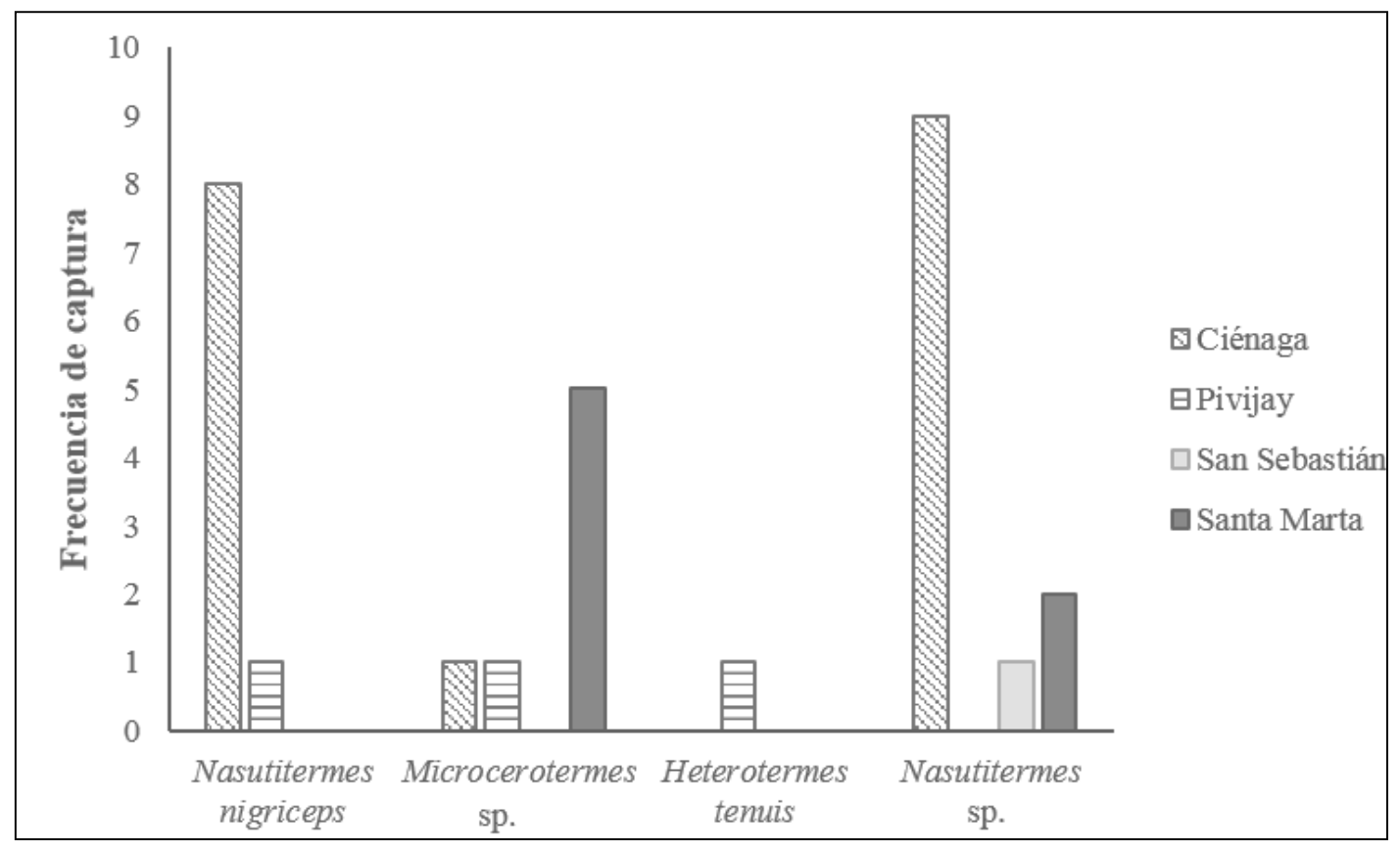

Figura 3. Frecuencia de termitas en municipios productores de mango del departamento del Magdalena. 


\section{DISCUSIÓN}

El género más abundante y más ampliamente distribuido en el departamento del Magdalena fue Nasutitermes (Figuras 2a-b y 3). Este género, con más de 244 especies descritas, se encuentra presente en 27 departamentos de Colombia, en altitudes entre 0 y $2800 \mathrm{~m}$ (Vargas-Niño et al., 2005) y posee especies que se han registrado previamente en cultivos de mango en otras regiones del mundo, como $N$. luzonicus en Filipinas (Acda, 2013), $N$. graveolus en el norte de Australia (Waite, 2002) y N. corniger, N. ephratae y N. nigriceps en Nicaragua (Maes, 2004). La morfoespecie más registrada en la mayoría de los huertos a lo largo del departamento del Magdalena fue Nasutitermes sp. (Figura 2b). La descripción de los soldados recolectados de esta morfoespecie coincide con las de Constantino (2002). Las especies de este género se pueden encontrar en nidos arbóreos y sus galerías transitan en troncos y ramas del árbol, alcanzando dimensiones de $2 \mathrm{~m}$ de altura y $1 \mathrm{~m}$ de ancho (Thorne et al., 1996; Pearce, 1997; Constantino, 2002; Arcila et al., 2013). En el caso de N. nigriceps, se encontró principalmente en la zona norte del departamento (Figura 2a).

Desde el punto de vista biológico algunas especies de Nasutitermes tienen adaptaciones. Thorne et al. (1996) y Gaju et al. (2015), evaluaron inclusiones o nódulos que se encuentran dentro de la galería de algunos nidos de termitas, concluyendo que utilizan los nódulos como una forma de almacenamiento de alimentos, debido a que encontraron que éstas poseen altas concentraciones de celulosa y bajas concentraciones de cutina, en comparación con un nido normal de este orden. Otra adaptación se relaciona con que $N$. nigriceps no almacena o cultiva su alimento, sino que lo procesa, es decir, tritura la comida y la incorpora a la matriz del nido para una utilización futura.

Se desconoce el impacto económico o el efecto de la asociación de estas especies con otros organismos en el desarrollo del cultivo de mango. Sin embargo, en otras especies vegetales, como Acacia mangium, se asume que pueden ocasionar daños indirectos y asociarse con otras especies cuando los árboles están debilitados 0 secos; por consiguiente, usualmente no se registra como plaga de importancia en la agricultura del país, sino que se considera secundaria a otros problemas sanitarios (Lores y Pinzón, 2011; Abadía et al., 2013).

Heterotermes tenuis (Figura 2d) solo se encontró en la zona centro del departamento. Esta especie es originaria 112 de Suramérica, aunque ha invadido las Antillas menores y el Caribe; se ha registrado en cultivos de caña de azúcar y eucalipto (Constantino, 2002; Szalanski et al., 2004). La morfología de los individuos recolectados coincidió con la descrita por Constantino (2002). Los soldados de $H$. tenuis son pequeños, la cabeza tiene forma casi rectangular, con mandíbulas generalmente grandes (alargados y sin dientes) casi rectas y visibles o aserradas; antenas con 15 - 18 antenómeros; pronoto casi plano o convexo; abdomen por lo general de color blanquecino y opaco con poros frontales y setas largas y cortas sobre su superficie (Constantino, 2002; Arcila et al., 2013). Los nidos de esta especie son subterráneos, así que la manera de detectar su presencia en los árboles es observando largos caminos de galerías que construyen en troncos y ramas (Arcila et al., 2013). Aunque se encuentran generalmente en árboles muertos y en descomposición (Arcila et al., 2013), durante los muestreos se observaron en plantas en pie, lo que puede deberse a que los agricultores de la zona sur no realizan podas sanitarias con frecuencia y se conservan árboles que pueden tener hasta 80 años de edad, con ramas secas y no productivas.

Los nidos de Microcerotermes sp. se encontraron en la zona centro y norte del departamento (Figura 2c), con presencia de sus nidos en los troncos o bases de los árboles. En cultivos como caña de azúcar usualmente se han registrado atacando raíces y tallos; también se han encontrado en tomate y algodón (Harris, 1954; Han y Ndiaye, 1998). Según Sermeño et al. (2003), los nidos de las especies de Microcerotermes son de conformación densa y dura, lo que los hace resistentes a la lluvia, solo permitiendo una ligera erosión de las capas más externas, mientras que las partes internas no se ven afectadas. Según Nickle et al. (1992), estos artrópodos pueden adaptar la consistencia de los nidos para una mayor protección en contra de las altas temperaturas de las zonas áridas y de los depredadores. Los soldados de este grupo son pequeños y con cabeza rectangular, tienen mandíbulas grandes aproximadamente simétricas, muy curvadas y sin dientes marginales, por lo general con margen serrado interno; labro angosto en el ápice y abdomen no blanquecino (Constantino, 2002). Especies de este género hasta el momento no han sido registradas como plaga común del cultivo de mango en este departamento, pero si se han documentado causando problemas en cultivos de eucalipto (Eucalyptus sp.) en el departamento del Magdalena (Gutiérrez et al., 2004; Gutiérrez et al., 2005).

Las diferencias en la distribución geográfica de las morfoespecies de termitas en cultivos de mango en el departamento del Magdalena no podrían ser atribuibles a 
un factor en particular, dado que la carencia de estudios en aspectos nutricionales, biológicos y adaptativos de las termitas no permiten hacer inferencias a partir de los datos obtenidos. En términos generales, las diferencias entre las tres regiones van desde la génesis de sus suelos hasta el manejo agronómico de los huertos. Por ejemplo, en la zona norte se observó durante este estudio un mayor desarrollo tecnológico en los cultivos del mango, en donde se realizan podas sanitarias, programas de fertilización completos y hay presencia de cultivos en grandes extensiones, a diferencia de la zona sur, en donde la mayor parte de los huertos están en traspatios y no tiene ningún manejo sanitario dado el bajo precio de compra del producto. En la zona centro las plantaciones tienen un mayor acompañamiento técnico que en la zona sur, pero la tecnificación aun es incipiente.

Además del manejo sanitario hay aspectos que requieren evaluación. Por ejemplo, algunos estudios han encontrado un mayor impacto de las termitas en zonas con abundante vegetación y con altas concentraciones de nutrientes. Además, dado que algunas especies de termitas utilizan selectivamente las partículas del suelo, zonas con suelos conformadas por partículas más finas para construir sus nidos (los cuales son subterráneos y/o epígeos) pueden favorecer su presencia, aun mas cuando se sabe que existen especies que requieren cantidades precisas de arena, limo y arcilla para la construcción de sus nidos y galerías (Lee et al., 1971; Jouquet et al., 2000). Para el caso de las zonas evaluadas en el departamento del Magdalena, la zona centro cuenta con suelos clase III, con frecuente encharcamiento, alto contenido de sales y fuerte erosión, lo que favorece la presencia de arcillas endurecidas y por ende ocasiona que las raíces de las plantas no profundicen mucho. Igualmente, la zona sur tiene suelos clase VIII, considerados no aprovechables para actividades agropecuarias por sus suelos pobres y pantanosos (Gobernación del Magdalena, 2008); adicionalmente, el Instituto Geográfico Agustín Codazzi IGAC (2009) ha realizado investigaciones sobre los tipos de suelos que existen en el departamento del Magdalena, indicando que la mayor parte de los suelos son aptas para el cultivo de $M$. indica, en especial la región norte, la cual comprende algunos sectores de Santa Marta y los municipios de Ciénaga y Zona Bananera, los cuales se visitaron para realizar este trabajo. Así mismo, el Instituto Geográfico Agustín Codazzi IGAC (2009) describe que los tipos de suelos que existen en el departamento del Magdalena son moderadamente profundos a muy profundos, bien a deficientemente drenados y con una fertilidad alta a moderada, originarios especialmente de depósitos aluviales, en donde predominan los suelos franco arenosos, franco arcillosos y arcillosos. A simple vista, no se infiere ninguna relación evidente entre los tipos de suelos y las morfoespecies de termitas asociadas, pero se requeriría realizar análisis de suelos en una escala espacial más pequeña en aquellos huertos con presencia permanente de termitas, a fin de determinar el rol del tipo de suelo en la distribución de estas morfoespecies.

\section{CONCLUSIONES}

Las termitas podrían llegar a ser uno de los problemas entomológicos más importantes en el departamento del Magdalena en lo que respecta a los cultivos de mango, por su alta prevalencia y amplia distribución.

Se requiere la evaluación de variables biológicas y su relación con factores edáficos y microambientales, a fin de disponer de herramientas para el diseño de posibles sistemas de control del insecto.

\section{AGRADECIMIENTOS}

Este proyecto fue parcialmente financiado por Colciencias y la Universidad del Magdalena en el marco del Plan de acompañamiento a Semilleros de investigación convocatoria 617-2013. Los autores agradecen a los propietarios y administradores de los huertos incluidos en la investigación por su gentil colaboración.

\section{BIBLIOGRAFÍA}

Abadía, J., Arcila, A. y Chacón, P. 2013. Incidencia y distribución de termitas (Isoptera) en cultivos de cítricos de la costa Caribe de Colombia. Revista Colombiana de Entomología 39(1): 1-8.

Abrol, D.P. 2015. Chapter 8: Tropical fruits. En: Abrol, D.P., Editor. Pollination Biology, Vol.1: Pests and pollinators of fruit crops. Springer International Publishing, Switzerland.

Acda, M.N. 2013. Geographical distribution of subterranean termites (Isoptera) in economically important regionsof Luzon, Philippines. Philippine Agricultural Scientist Journal 96(2): 205-209.

ANACAFE. 2004. Cultivo de Hule. Programa de diversificación de ingresos de la empresa cafetalera, Guatemala. 
Arcila, A., Abadía, J.C, Achury R.A., Carrascal, F.F. y Yacomelo, M.J. 2013. Manual para la identificación y manejo de termitas y otros insectos plagas de los cítricos en la región Caribe de Colombia. Corpoica, Colombia.

Bignell, D. E., Roisin, Y. y Lo, N. 2011. Biology of Termites: a Modern Synthesis. Springer, Dordrecht, The Netherlands.

Corpoica- Corporación Colombiana de Investigaciones Agropecuarias y Asohofrucol- Asociación Hortifrutícola de Colombia. 2013. Modelo Tecnológico para el cultivo del mango en el Valle del alto Magdalena en el Departamento del Tolima. Ministerio de Agriculturay Desarrollo Rural, Bogotá.

Cartagena, J.R. y Vega, D. 1992. Fruticultura colombiana, el mango, Manual de asistencia técnica $N^{\circ} 43$. PM Ediciones, Bogotá.

Constantino, R. 2002. An illustrated key to Neotropical termite genera (Insecta:Isoptera) based primarily on soldiers. Zootaxa 67: 1-40.

Gaju, M., Bach, C. y Molero, R. 2015. Orden Isoptera. Revista IDEA-SEA 49:1-17.

Galán, V. 1999. El Cultivo Del Mango. Ediciones Mundi Prensa, Madrid.

Gobernación del Magdalena. 2008. Magdalena, Plan de desarrollo departamental 2008-2011. Gobernación del Magdalena. Santa Marta.

Gutiérrez, A.I., Uribe, S. y Quiroz, J. A. 2004. Termitas asociadas a plantaciones de Eucalyptus spp. en una reforestadora en Magdalena, Colombia. Manejo Integrado de Plagas y Agroecología (Costa Rica) 72: 54-59.

Gutiérrez, A.I., Saldarriaga, Y., Uribe, S., Zuluaga, M. y Pineda, F. 2005. Patogenicidad de Paecilomyces lilacinus y Metarhizium anisopliae sobre termitas Microcerotermes sp. (Isoptera: Termitidae). Revista Colombiana de Entomología 31(1): 9-14.

Han, S.H. y Ndiaye, A.B. 1998. Dégâts causés par les termites (Isoptera) sur les arbres fruitiers dans la region de Dakar (Sénégal). Actes des Colloques Insectes Sociaux 10:111-117.

Harris, W.V. 1954. Termites and tropical agriculture. Tropical Agriculture (Trinidad) 31:11-18.

Hill, D.S. 2008. Major tropical crops and their pest spectra En: Hill, D.S., Editor. Pests of Crops in Warmer Climates and Their Control. Springer, Netherlands.
Instituto Geográfico Agustín Codazzi IGAC. 2009. Estudio general de suelos y zonificación de tierras departamento del Magdalena, escala 1:100.000. Imprenta Nacional de Colombia, Bogotá.

Iqbal, N., Khan, H.A. y Saeed, S. 2015. Response of Microtermes mycophagus (Isoptera: Termitidae) to twenty one wood species. PeerJ 3:e1132.

Jouquet, P., Lepage, M. y Velde, B. 2000. Termite soil preferences and particle selections: trategies related to ecological requirements. Insectes Sociaux 49: 1-7.

Kannan, M. y Venugopala, N. 2006. Seasonal incidence and population fluctuation of dipteran, isopteran, hymenopteran and thysanopteran pest of mango. Journal of Plant Protection and Environment 3(2): 50-55.

Krishna, K., Grimaldi, D.A., Krishna, V. y Engel, M. 2013a. Treatise of the Isoptera of the world. 1. Introduction. AMNH Bulletin 377 (1):1-202.

Krishna, K., Grimaldi, D.A., Krishna, V. y Engel, M. 2013b. Treatise of the Isoptera of the world. 2. Basal families. AMNH Bulletin 377 (2): 201-624.

Kondo-R., D.T. 2010. III. Insectos. En: Bernal, J.A. y Díaz, C.A., Editores. Tecnología para el cultivo de mango. Manual Técnico. Produmedios, Bogotá.

Lee, K.E. y Wood, T.G. 1971. Physical and chemical effects on soils of some Australian termites, and their pedological signicance. Pedobiologia 11: 376 - 409.

Lores, A. y Pinzón, O. 2011. Insectos fitófagos en plantaciones comerciales de Acacia mangium Willd. en la costa Atlántica y la Orinoquia colombiana. Colombia Forestal 14 (2):175-188.

Maes, J.M. 2004. Insectos asociados a algunos cultivos tropicales en el Atlántico de Nicaragua. Parte XI. Mango (Mangifera indica, Anacardiaceae). Revista Nicaragüense de Entomología 64: Supplemento 1, parte XI: 1-158.

Nickle, D. y Collins, M. 1992. The Termites of Panama (Isoptera) En: Quintero, D. y Aiello, A. Editores. Insects of Panama and Mesoamerica: selected studies. Oxford University Press, New York.

Pearce, M. 1997. Termites: biology and pest management. CAB international, New York. 
Rogers, L., French, J. y Elgar M. 1999. Suppression of plant growth on the mounds of the termite Coptotermes lacteus Froggatt (Isoptera: Rhinotermitidae). Insectes Sociaux 46: 366-371.

Sermeño, J., Jones, D., Menjivar, D., Paniagua M. y Monro, A. 2003. Termitas de los cafetales de El Salvador. Facultad de Ciencias Agronómicas Universidad de El Salvador._URL: http://ri.ues.edu.sv/9080/1/A \%20Termitas \% 20de \% 20 los $\% 20$ cafetales $\% 20$ de $\% 20$ El \%20Salvador \%2C\%202003. pdf. Consultado: 26 de agosto 2017.

Szalanski, A.L, Scheffrahn, R.H., Austin, J.W., Krecek, J. y $\mathrm{Su}, \mathrm{N}-\mathrm{Y}$. 2004. Molecular phylogeny and biogeography of Heterotermes (Isoptera: Rhinotermitidae) in the West Indies. Annals of the Entomological Society of America 97: 574-585.
Thorne, B., Collins, M. y Bjorndal K. 1996. Architecture and nutrient analysis of arboreal carton nests of two neotropical Nasutitermes species (Isoptera: Termitidae), with notes on embedded nodules. Florida Entomologist 79(1): 27-36.

Vargas-Niño, A.P., Sánchez-Muñoz, O.D. y SernaCardona, F.J. 2005. Lista de los géneros de Termitidae (Insecta: Isoptera) de Colombia. Biota Colombiana 6(2): 181-190.

Waite, G.K. 2002. Pest and pollinators of mango. En: Peña, J., Sharp, J.L. y Wysoki, M., Editores. Tropical Fruit Pests and Pollinators: Biology, Economic Importance, Natural Enemies and Control. Cabi Publishing. London.

Fecha de recepción: 31/03/2017

Fecha de aceptación: 01/09/2017

Publicado en línea: 14/09/2017

Para citar este artículo: Hurtado-Borrero, H.Y. Manga-Candelario, D.A. y SepúlvedaCano, P.A. 2017. Registro de termitas (Isoptera) asociadas a cultivos de mango (Mangifera indica) en el departamento del Magdalena, Colombia. Intropica 12(2): 109-115. DOI: http://dx.doi.org/10.21676/23897864.2286 